\title{
A Table of Elliptic Integrals of the Third Kind*
}

\author{
By B. C. Carlson
}

\begin{abstract}
As many as 72 elliptic integrals of the third kind in previous tables are unified by evaluation in terms of $R$-functions instead of Legendre's integrals. The present table includes only integrals having integrands with real singular points. In addition to 31 integrals of the third kind, most of them unavailable elsewhere, 10 integrals of the first and second kinds from an earlier table are listed again in new notation. In contrast to conventional tables, the interval of integration is not required to begin or end at a singular point of the integrand. Fortran codes for the standard $R$-functions $R_{C}$ and $R_{J}$, revised to include their Cauchy principal values, are listed in a Supplement.
\end{abstract}

1. Introduction. A table [4] of elliptic integrals of the first and second kinds is extended in this paper to integrals of the third kind. We choose a standard form,

$$
[p]=\left[p_{1}, \ldots, p_{n}\right]=\int_{y}^{x} \prod_{i=1}^{n}\left(a_{i}+b_{i} t\right)^{p_{i} / 2} d t
$$

where $p_{1}, \ldots, p_{n}$ are integers and the integrand is real. The integral is assumed to be well defined; in particular, if $p_{i}$ is odd, $a_{i}+b_{i} t$ must be positive in the open interval of integration. Many integrals like

$$
\int\left(1+n \sin ^{2} \phi\right)^{-1}\left(1-k^{2} \sin ^{2} \phi\right)^{-1 / 2} d \phi
$$

and

$$
\int\left(1+n z^{2}\right)^{-1}\left[\left(1-z^{2}\right)\left(1-k^{2} z^{2}\right)\right]^{-1 / 2} d z
$$

can be put in the form (1.1) by letting $t=\sin ^{2} \phi$ or $t=z^{2}$.

If we assume the b's are nonzero and no two of the quantities $a_{i}+b_{i} t$ are proportional, then $\left[p_{1}, \ldots, p_{n}\right]$ is an elliptic integral if the number of odd $p$ 's is exactly three (the "cubic case") or four (the "quartic case"). It is elliptic of third kind if at least one of the $p$ 's is even and negative or if $p_{1}+\cdots+p_{n}=-2,0,2,4, \ldots$, the latter condition being impossible in the cubic case. Otherwise, it is first or second kind, and the only integrals of first kind are $[-1,-1,-1]$ and $[-1,-1,-1,-1]$.

The conditions that the integral be of third kind are deduced by using $[2,(8.1-2)]$ to express $[p]$ as an $R$-function with " $b$-parameters" $-p_{1} / 2, \ldots,-p_{n} / 2,2+\sum p_{i} / 2$. The integral is of third kind if at least one of these parameters is a positive integer (see $[2, \S 8.5, \S 9.2])$.

Received October 14, 1987.

1980 Mathematics Subject Classification (1985 Revision). Primary 33A25; Secondary 33A30.

*Part of this work was done at the University of Maryland, where the author was a visitor at the Institute for Physical Science and Technology, with the support of AROD contract DAAG 2980-C-0032. The rest was done in the Ames Laboratory, which is operated for the U.S. Department of Energy by Iowa State University under contract no. W-7405-ENG-82. The work was supported by the Director of Energy Research, Office of Basic Energy Sciences.

(C) 1988 American Mathematical Society $0025-5718 / 88 \$ 1.00+\$ .25$ per page 
We shall replace Legendre's integrals of the first and third kinds by

$$
\begin{gathered}
R_{F}(x, y, z)=\frac{1}{2} \int_{0}^{\infty}[(t+x)(t+y)(t+z)]^{-1 / 2} / d t \\
R_{J}(x, y, z, \rho)=\frac{3}{2} \int_{0}^{\infty}[(t+x)(t+y)(t+z)]^{-1 / 2}(t+\rho)^{-1} d t .
\end{gathered}
$$

Besides being symmetric in $x, y, z$, these $R$-functions are homogeneous:

$$
\begin{gathered}
R_{F}(\lambda x, \lambda y, \lambda z)=\lambda^{-1 / 2} R_{F}(x, y, z), \\
R_{J}(\lambda x, \lambda y, \lambda z, \lambda \rho)=\lambda^{-3 / 2} R_{J}(x, y, z, \rho),
\end{gathered}
$$

and they are normalized so that

$$
R_{F}(x, x, x)=x^{-1 / 2}, \quad R_{J}(x, x, x, x)=x^{-3 / 2} .
$$

Two special cases are denoted by

$$
R_{C}(x, y)=R_{F}(x, y, y), \quad R_{D}(x, y, z)=R_{J}(x, y, z, z) .
$$

The function $R_{D}$ replaces Legendre's integral of the second kind, and $R_{C}$ is an elementary function** embracing the logarithmic, inverse circular, and inverse hyperbolic functions [3, (4.9)-(4.13)]. Use of $R_{C}$ allows unification of formulas for circular and hyperbolic cases of integrals of the third kind.

Fortran codes [5] for $R_{C}$ and $R_{J}$ are listed in the Supplements section of this issue, and codes for $R_{F}$ and $R_{D}$ are given in a Supplement to [4]. All four can be found also in most of the major software libraries, but the codes for $R_{C}$ and $R_{J}$ in the Supplement have recently been modified to compute the Cauchy principal value of the integral when the last variable is negative. As shown in Sections 5 and 6 , Cauchy principal values are sometimes needed when using the formulas of this paper.

As explained in [4], the present table differs from customary integral tables $[1],[7]^{* * *},[8]$ in two respects: We do not require the interval of integration to begin or end at a branch point of the integrand, and we do not separate special cases according to the positions of the branch points relative to the interval of integration and to one another. The unification of these special cases is made possible by application of the addition theorem and by the use of $R$-functions instead of Legendre's integrals. The present table includes only integrands with real branch points; conjugate complex branch points resulting from an irreducible polynomial $a_{i}+b_{i} t+c_{i} t^{2}$ are deferred to a later paper.

The table in Section 2 consists of quartic cases, since many cubic cases are included in these by taking $a_{i}=1$ and $b_{i}=0$ for various values of $i$. To select integrals that are relatively simple, we arbitrarily require $\sum\left|p_{i}\right| \leq 8$ and $\sum p_{i} \leq$ 0 . Apart from permutation of subscripts in (1.1), there are just 37 quartic cases satisfying these criteria. We omit nine with $p_{5}= \pm 2, p_{6}= \pm 2$ since they can

**Although not in connection with elliptic integrals, the use of a function equivalent to the reciprocal of $R_{C}$ was proposed in 1897 by Fubini, while still a student at Pisa, in his first published paper [6]. I am obliged to Professor Luigi Gatteschi for this reference.

*** Tables 3 and 4 in [7] use Legendre's $\Pi$ as defined by [1, (110.04)] and are inconsistent with $[7,8.111(4)]$, which differs in the sign of the parameter. The same is true of the 1965 edition. 
easily be reduced to the others by $(2.51)-(2.53)$, but we add three with $\sum p_{i}>$ 0 : $[1,1,1,-1],[1,1,1,1]$, and $[1,1,1,1,-2]$. Thus we list 31 quartic cases, most of which are not available in previous tables. The formulas for $[1,-1,-1,-1]$, $[1,1,-1,-3],[1,1,-1,-1]$, and $[1,1,1,-1]$ respectively unify $32,72,48$, and 32 special cases in Byrd and Friedman's table [1].

All cubic cases of third kind with $\sum\left|p_{i}\right| \leq 7$ are contained in the quartic cases of Section 2 with three exceptions: $[1,1,1,-2],[3,1,-1,-2]$, and $[3,1,1,-2]$. Cubic cases of all three kinds will be discussed in a later paper.

Because some of the formulas are cumbersome when written out in the style of [4], we have introduced abbreviations to save space. For uniformity of style, the quartic cases of first and second kinds listed in [4] are listed again in Section 3 in the new notation. Derivation of formulas by recurrence relations is discussed in Section 4. The fundamental formula for $[1,-1,-1,-1,-2]$ is proved in Section 5 , and Cauchy principal values are discussed in Section 6 . All integral formulas have been checked by numerical integration; some details of the checks are given in the Supplement.

2. Table of Quartic Cases. We assume $x>y$ and $a_{i}+b_{i} t>0, y<t<x$, for $i=1, \ldots, 4$. Assumptions about $a_{5}+b_{5} t$ will be stated where necessary. We define

$$
\begin{gathered}
d_{i j}=a_{i} b_{j}-a_{j} b_{i}, \quad r_{i j}=\frac{d_{i j}}{b_{i} b_{j}}=\frac{a_{i}}{b_{i}}-\frac{a_{j}}{b_{j}} \\
X_{i}=\left(a_{i}+b_{i} x\right)^{1 / 2}, \quad Y_{i}=\left(a_{i}+b_{i} y\right)^{1 / 2} \\
U_{i j}=\left(X_{i} X_{j} Y_{k} Y_{m}+Y_{i} Y_{j} X_{k} X_{m}\right) /(x-y)
\end{gathered}
$$

where $i, j, k, m$ is any permutation of $1,2,3,4$;

$$
\begin{gathered}
W^{2}=U_{12}^{2}-d_{13} d_{14} d_{25} / d_{15} \\
Q^{2}=\left(X_{5} Y_{5} W / X_{1} Y_{1}\right)^{2}, \quad P^{2}=Q^{2}+d_{25} d_{35} d_{45} / d_{15} \\
A\left(p_{1}, \ldots, p_{n}\right)=X_{1}^{p_{1}} \cdots X_{n}^{p_{n}}-Y_{1}^{p_{1}} \cdots Y_{n}^{p_{n}}
\end{gathered}
$$

These definitions imply, if $P$ is chosen positive,

$$
\begin{gathered}
U_{i j}^{2}-U_{i k}^{2}=d_{i m} d_{j k}, \\
P=\left(X_{1}^{-1} X_{2} X_{3} X_{4} Y_{5}^{2}+Y_{1}^{-1} Y_{2} Y_{3} Y_{4} X_{5}^{2}\right) /(x-y),
\end{gathered}
$$

and hence, with the help of $[4,(5.22)]$,

$$
\begin{gathered}
U_{13}^{2}=U_{12}^{2}-d_{14} d_{23}, \quad U_{14}^{2}=U_{12}^{2}-d_{13} d_{24}, \\
W^{2}=U_{1 i}^{2}-d_{1 j} d_{1 k} d_{i 5} / d_{15},
\end{gathered}
$$

where $i, j, k$ is any permutation of $2,3,4$. When $a_{5}=1$ and $b_{5}=0$, the quantities $W, P, Q$ become $W_{1}, P_{1}, Q_{1}$ :

$$
\begin{gathered}
W_{1}^{2}=U_{12}^{2}-b_{2} d_{13} d_{14} / b_{1}, \\
Q_{1}^{2}=\left(W_{1} / X_{1} Y_{1}\right)^{2}, \quad P_{1}^{2}=Q_{1}^{2}+b_{2} b_{3} b_{4} / b_{1} .
\end{gathered}
$$

If one limit of integration is infinite, (2.3) simplifies to

$$
\begin{array}{ll}
U_{i j}=\left(b_{i} b_{j}\right)^{1 / 2} Y_{k} Y_{m}+Y_{i} Y_{j}\left(b_{k} b_{m}\right)^{1 / 2}, & x=+\infty \\
U_{i j}=X_{i} X_{j}\left(b_{k} b_{m}\right)^{1 / 2}+\left(b_{i} b_{j}\right)^{1 / 2} X_{k} X_{m}, & y=-\infty
\end{array}
$$


all square roots being nonnegative, while

$$
\begin{array}{ll}
Q^{2}=\left(b_{5} / b_{1}\right)\left(Y_{5} W / Y_{1}\right)^{2}, & x=+\infty, \\
Q^{2}=\left(b_{5} / b_{1}\right)\left(X_{5} W / X_{1}\right)^{2}, & y=-\infty .
\end{array}
$$

All quartic cases will be expressed in terms of the quantities

$$
\begin{aligned}
& I_{1}=2 R_{F}\left(U_{12}^{2}, U_{13}^{2}, U_{14}^{2}\right) \\
& I_{2}=\frac{2}{3} d_{12} d_{13} R_{D}\left(U_{12}^{2}, U_{13}^{2}, U_{14}^{2}\right)+\frac{2 X_{1} Y_{1}}{X_{4} Y_{4} U_{14}}, \\
& I_{3}=\frac{2 d_{12} d_{13} d_{14}}{3 d_{15}} R_{J}\left(U_{12}^{2}, U_{13}^{2}, U_{14}^{2}, W^{2}\right)+2 R_{C}\left(P^{2}, Q^{2}\right) \\
& I_{3}^{\prime}=\frac{-2 d_{12} d_{13} d_{14}}{3 b_{1}} R_{J}\left(U_{12}^{2}, U_{13}^{2}, U_{14}^{2}, W_{1}^{2}\right)+2 R_{C}\left(P_{1}^{2}, Q_{1}^{2}\right) .
\end{aligned}
$$

It will be seen from the tables that

$$
\begin{array}{ll}
I_{1}=[-1,-1,-1,-1], & I_{2}=[1,-1,-1,-3], \\
I_{3}=[1,-1,-1,-1,-2], & I_{3}^{\prime}=[1,-1,-1,-1] .
\end{array}
$$

Thus, $I_{3}$ reduces to $I_{3}^{\prime}$ if $a_{5}=1$ and $b_{5}=0$, and $I_{3}$ reduces to $I_{2}$ if $a_{5}=a_{4}$ and $b_{5}=b_{4}$.

If one limit of integration is a branch point of the integrand, then $X_{i}$ or $Y_{i}$ is 0 for some value of $i \leq 4$, and one of the two terms on the right-hand side of (2.3) vanishes. If $X_{1} Y_{1}=0$ then $P, Q, P_{1}$, and $Q_{1}$ are infinite, and the $R_{C}$-functions in (2.15) and (2.16) vanish by (1.6) and (1.4). If both limits of integration are branch points, the elliptic integral is called complete, and $U_{12} U_{13} U_{14}=0$. It is not assumed that $b_{i} \neq 0$ nor that $d_{i j} \neq 0$, unless one of these quantities occurs in a denominator. The relation $d_{i j}=0$ is equivalent to proportionality of $a_{i}+b_{i} t$ and $a_{j}+b_{j} t$.

We are now ready to list 31 cases of

$$
\left[p_{1}, \ldots, p_{5}\right]=\int_{y}^{x}\left(a_{1}+b_{1} t\right)^{p_{1} / 2} \cdots\left(a_{5}+b_{5} t\right)^{p_{5} / 2} d t
$$

15 with $p_{5}=0,2$, or 4 and 16 with $p_{5}=-2$ or -4 . The first 10 have $p_{5}=0$ and involve $I_{3}^{\prime}$ but not $I_{3}$.

$$
\begin{gathered}
{[1,-1,-1,-1]=I_{3}^{\prime} .} \\
{[1,1,-1,-3]=\left(b_{2} I_{3}^{\prime}+d_{24} I_{2}\right) / b_{4} .} \\
{[3,-1,-1,-3]=\left(b_{1} I_{3}^{\prime}+d_{14} I_{2}\right) / b_{4} .} \\
{[1,1-1,-1]=\left[b_{2}\left(r_{13}+r_{24}\right) I_{3}^{\prime}+d_{24} r_{34} I_{2}-d_{12} r_{13} I_{1}\right] / 2} \\
+A(1,1,1,-1) / b_{3} . \\
{[3,-1,-1,-1]=\left(b_{1} / 2\right)\left[\sum_{i=2}^{4} r_{1 i} I_{3}^{\prime}+b_{4} r_{24} r_{34} I_{2}\right.} \\
\left.-b_{1} r_{12} r_{13} I_{1}+2 A(1,1,1,-1) / b_{2} b_{3}\right] .
\end{gathered}
$$




$$
\begin{aligned}
& {[1,1,1,-3]=\left(b_{2} b_{3} / 2 b_{4}\right)\left[\sum_{i=1}^{3} r_{i 4} I_{3}^{\prime}+3 b_{4} r_{24} r_{34} I_{2}-b_{1} r_{12} r_{13} I_{1}\right]} \\
& +A(1,1,1,-1) / b_{4} \text {. } \\
& {[3,1,-1,-3]=\left(b_{1} b_{2} / 2 b_{4}\right)\left[\left(r_{13}+2 r_{14}+r_{24}\right) I_{3}^{\prime}\right.} \\
& \left.+b_{4} r_{24}\left(2 r_{14}+r_{34}\right) I_{2}-b_{1} r_{12} r_{13} I_{1}\right] \\
& +\left(b_{1} / b_{3} b_{4}\right) A(1,1,1,-1) \text {. } \\
& {[1,1,1,-5]=\left(b_{2} b_{3} / b_{4}^{2}\right) I_{3}^{\prime}+\left(d_{24} d_{34} / 3 b_{4}^{3}\right) \sum_{i=1}^{3} r_{i 4}^{-1} I_{2}} \\
& -\left(d_{12} d_{13} / 3 b_{4} d_{14}\right) I_{1}-\left(2 / 3 b_{4}\right) A(1,1,1,-3) \text {. } \\
& {[1,1,1,-1]=\left(b_{2} b_{3} / 8\right)\left(r_{14}^{2}-r_{12}^{2}-r_{13}^{2}+2 r_{24} r_{34}\right) I_{3}^{\prime}} \\
& +\left(\sum_{i=1}^{3} r_{i 4} / 8\right)\left[b_{3} d_{24} r_{34} I_{2}+2 A(1,1,1,-1)\right] \\
& +\left(b_{3} d_{12} r_{13} / 8\right)\left(r_{12}-r_{34}\right) I_{1}+A(1,1,1,1) / 2 b_{4} \text {. } \\
& {[1,1,1,1]=\left(b_{2} b_{3} b_{4} / 16\right)\left(r_{12}-r_{34}\right)\left(r_{14}^{2}-r_{23}^{2}\right) I_{3}^{\prime}} \\
& -\left[\left(r_{12}-r_{34}\right)^{2}+2\left(r_{14}^{2}+r_{23}^{2}\right)\right] \\
& \text { · }\left[d_{24} d_{34} I_{2}+2 b_{4} A(1,1,1-1)\right] / 48 \\
& +\left(b_{3} b_{4} d_{12} r_{13} / 48\right)\left(4 r_{24} r_{34}-3 r_{14}^{2}+3 r_{23}^{2}\right) I_{1} \\
& -\left(\sum_{i=2}^{4} r_{1 i} / 12\right) A(1,1,1,1)+A(3,1,1,1) / 3 b_{1} \text {. }
\end{aligned}
$$

The next five integrals, with $p_{5}=2$ or 4 , involve $I_{3}^{\prime}$ but not $I_{3}$. No restriction is placed on $a_{5}$ or $b_{5}$.

$$
\begin{gathered}
{[-1,-1,-1,-1,2]=\left(b_{5} I_{3}^{\prime}-d_{15} I_{1}\right) / b_{1} .} \\
{[1,-1,-1,-1,2]=\left(b_{5} / 2\right)\left[\left(r_{12}-r_{35}-r_{45}\right) I_{3}^{\prime}+b_{4} r_{24} r_{34} I_{2}\right.} \\
\left.-b_{1} r_{12} r_{13} I_{1}+\left(2 / b_{2} b_{3}\right) A(1,1,1,-1)\right] . \\
{[1,-1,-1,-3,2]=\left(b_{5} I_{3}^{\prime}-d_{45} I_{2}\right) / b_{4} .} \\
{[1,1,-1,-3,2]=\left(b_{2} b_{5} / 2 b_{4}\right)\left[\left(r_{13}+r_{24}-2 r_{45}\right) I_{3}^{\prime}\right.} \\
\left.+b_{4} r_{24}\left(r_{34}-2 r_{45}\right) I_{2}-b_{1} r_{12} r_{13} I_{1}\right] \\
+\left(b_{5} / b_{3} b_{4}\right) A(1,1,1,-1) . \\
=\left(b_{5}^{2} / 2 b_{1}\right)\left[-\sum_{i=1}^{4} r_{i 5} I_{3}^{\prime}+b_{4} r_{24} r_{34} I_{2}+b_{1}\left(2 r_{15}^{2}-r_{12} r_{13}\right) I_{1}\right. \\
\left.+\left(2 / b_{2} b_{3}\right) A(1,1,1,-1)\right]
\end{gathered}
$$


The next 11 integrals have $p_{5}=-2$, and we assume $a_{5}+b_{5} t$ either is positive on the closed interval of integration or changes sign in the open interval of integration. In the latter case the integral is interpreted as a Cauchy principal value; see Section 6. All 11 integrals involve $I_{3}$, and the six with $\sum p_{i} \geq-2$ involve $I_{3}^{\prime} / b_{5}$ also.

$$
\begin{aligned}
& {[1,-1,-1,-1,-2]=I_{3} .} \\
& {[-1,-1,-1,-1,-2]=\left(b_{5} I_{3}-b_{1} I_{1}\right) / d_{15} \text {. }} \\
& {[1,1,-1,-1,-2]=\left(d_{25} I_{3}+b_{2} I_{3}^{\prime}\right) / b_{5} \text {. }} \\
& {[1,-1,-1,-3,-2]=\left(b_{5} I_{3}-b_{4} I_{2}\right) / d_{45} \text {. }} \\
& {[1,1,-1,-3,-2]=\left(d_{25} I_{3}-d_{24} I_{2}\right) / d_{45} \text {. }} \\
& {[-1,-1,-1,-3,-2]=\left(b_{5}^{2} / d_{15} d_{45}\right) I_{3}-\left(b_{4}^{2} / d_{14} d_{45}\right) I_{2}} \\
& +\left(b_{1}^{2} / d_{14} d_{15}\right) I_{1} \text {. } \\
& {[1,1,1,-3,-2]=\left(d_{25} d_{35} / b_{5} d_{45}\right) I_{3}+\left(b_{2} b_{3} / b_{4} b_{5}\right) I_{3}^{\prime}} \\
& -\left(d_{24} d_{34} / b_{4} d_{45}\right) I_{2} \text {. } \\
& {[3,-1,-1,-1,-2]=\left(d_{15} I_{3}+b_{1} I_{3}^{\prime}\right) / b_{5}} \\
& {[3,1,-1,-1,-2]=\left(d_{15} d_{25} / b_{5}^{2}\right) I_{3}+\left(b_{1} b_{2} / 2 b_{5}\right)} \\
& \cdot\left[\left(r_{13}+2 r_{15}+r_{24}\right) I_{3}^{\prime}+b_{4} r_{24} r_{34} I_{2}-b_{1} r_{12} r_{13} I_{1}\right] \\
& +\left(b_{1} / b_{3} b_{5}\right) A(1,1,1,-1) \text {. } \\
& {[1,1,1,-1,-2]=\left(d_{25} d_{35} / b_{5}^{2}\right) I_{3}+\left(b_{2} b_{3} / 2 b_{5}\right)} \\
& \cdot\left[\left(r_{15}+r_{35}+r_{24}\right) I_{3}^{\prime}+b_{4} r_{24} r_{34} I_{2}-b_{1} r_{12} r_{13} I_{1}\right] \\
& +A(1,1,1,-1) / b_{5} \text {. } \\
& {[1,1,1,1,-2]=\left(d_{25} d_{35} d_{45} / b_{5}^{3}\right) I_{3}+\left(b_{2} b_{3} b_{4} / 8 b_{5}\right)\left(\sigma^{2}-2 \tau\right) I_{3}^{\prime}} \\
& +\left(\sigma / 8 b_{5}\right)\left[d_{24} d_{34} I_{2}+2 b_{4} A(1,1,1,-1)\right] \\
& +\left(b_{4} d_{12} d_{13} / 8 b_{1} b_{5}\right)\left(r_{12}-r_{35}-3 r_{45}\right) I_{1} \\
& +A(1,1,1,1) / 2 b_{5}
\end{aligned}
$$

where

$$
\sigma=\sum_{i=1}^{4} r_{i 5}, \quad \tau=\sum_{i=1}^{4} r_{i 5}^{2} .
$$

The final five integrals have $p_{5}=-4$. All five involve $I_{3}$, and the two with $\sum p_{i} \geq-2$ involve $I_{3}^{\prime} / b_{5}^{2}$ also. We assume $a_{5}+b_{5} t$ is positive on the closed interval of integration.

$$
\begin{aligned}
{[1,1,-1,-1,-4]=} & \frac{1}{2}\left(\frac{d_{23}}{d_{53}}+\frac{d_{24}}{d_{54}}-\frac{d_{21}}{d_{51}}\right) I_{3}+\frac{d_{24} d_{34}}{2 d_{35} d_{45}} I_{2} \\
& -\frac{d_{12} d_{13}}{2 d_{15} d_{35}} I_{1}-\frac{1}{d_{35}} A(1,1,1,-1,-2)
\end{aligned}
$$




$$
\begin{aligned}
& {[1,-1,-1,-1,-4]=\frac{1}{2}\left(\frac{b_{1}}{d_{15}}-\sum_{i=2}^{4} \frac{b_{i}}{d_{i 5}}\right) I_{3}+\frac{b_{5} d_{24} d_{34}}{2 d_{25} d_{35} d_{45}} I_{2}} \\
& -\frac{b_{5} d_{12} d_{13}}{2 d_{15} d_{25} d_{35}} I_{1}-\frac{b_{5}}{d_{25} d_{35}} A(1,1,1,-1,-2) \text {. } \\
& {[1,1,1,-1,-4]=\left(b_{2} b_{3} / 2 b_{5}^{2}\right)\left[b_{5}\left(r_{25}+r_{25} r_{35} / r_{15}-r_{24} r_{35} / r_{45}\right) I_{3}\right.} \\
& \left.+2 I_{3}^{\prime}+\left(b_{4} r_{24} r_{34} / r_{45}\right) I_{2}-\left(b_{1} r_{12} r_{13} / r_{15}\right) I_{1}\right] \\
& \text { - } A(1,1,1,-1,-2) / b_{5} \text {. } \\
& {[-1,-1,-1,-1,-4]=\frac{-1}{2 d_{15}} \sum_{i=1}^{4} \frac{1}{r_{i 5}} I_{3}+\frac{b_{5}^{2} d_{24} d_{34}}{2 d_{15} d_{25} d_{35} d_{45}} I_{2}} \\
& +\frac{b_{1}^{2}}{d_{15}^{2}}\left(1-\frac{r_{12} r_{13}}{2 r_{25} r_{35}}\right) I_{1} \\
& -\frac{b_{5}^{2}}{d_{15} d_{25} d_{35}} A(1,1,1,-1,-2) \text {. } \\
& {[1,1,1,1,-4]=\left(d_{25} d_{35} d_{45} / 2 b_{5}^{4}\right) \sum_{i=1}^{4} r_{i 5}^{-1} I_{3}} \\
& +\left(b_{2} b_{3} b_{4} / 2 b_{5}^{2}\right) \sum_{i=1}^{4} r_{i 5} I_{3}^{\prime}+\left(d_{24} d_{34} / b_{5}^{2}\right) I_{2} \\
& -\left(b_{4} d_{12} d_{13} / 2 b_{5} d_{15}\right)\left(r_{15}+r_{45}\right) I_{1} \\
& \text { - } A(1,1,1,1,-2) / b_{5}+\left(2 b_{4} / b_{5}^{2}\right) A(1,1,1,-1) \text {. }
\end{aligned}
$$

The nine integrals with $p_{5}= \pm 2, p_{6}= \pm 2, \sum\left|p_{i}\right| \leq 8$, and $\sum p_{i} \leq 0$ can be reduced to those listed above by using the identities

$$
\begin{gathered}
\left(a_{5}+b_{5} t\right)^{-1}\left(a_{6}+b_{6} t\right)^{-1}=\left[b_{5}\left(a_{5}+b_{5} t\right)^{-1}-b_{6}\left(a_{6}+b_{6} t\right)^{-1}\right] / d_{65} \\
\left(a_{5}+b_{5} t\right)^{-1}\left(a_{6}+b_{6} t\right)=\left[b_{6}+d_{65}\left(a_{5}+b_{5} t\right)^{-1}\right] / b_{5} \\
\left(a_{5}+b_{5} t\right)\left(a_{6}+b_{6} t\right)=\left[d_{65}\left(a_{5}+b_{5} t\right)+b_{6}\left(a_{5}+b_{5} t\right)^{2}\right] / b_{5}
\end{gathered}
$$

3. Quartic Cases of First or Second Kind. In [4] the nine quartic integrals of first or second kind with $\sum\left|p_{i}\right| \leq 8$ were evaluated in terms of $R_{F}$ and $R_{D}$. Since $R_{D}$ is symmetric in only its first two variables, the third was chosen variously as $U_{12}^{2}, U_{13}^{2}$, or $U_{14}^{2}$ to simplify the formulas. In (2.14) and hence throughout Section $2, U_{14}^{2}$ is the choice. For uniformity of notation and for use in deriving the formulas of Section 2, we list again the nine integrals in present notation and add for convenience $[-1,-1,-1,-3]$, which is a special case of $[-1,-1,-1,-3,2]$.

$$
\begin{gathered}
{[-1,-1,-1,-1]=I_{1} .} \\
{[1,-1,-1,-3]=I_{2} .} \\
{[-1,-1,-1,-3]=\left(b_{4} I_{2}-b_{1} I_{1}\right) / d_{14} .} \\
{[-1,-1,-1,-3,2]=\left(d_{54} I_{2}+d_{15} I_{1}\right) / d_{14} .} \\
{[1,1,-3,-3]=\left[2 d_{24} I_{2}+d_{12} I_{1}+2 A(1,1,-1,-1)\right] / d_{34}}
\end{gathered}
$$




$$
\begin{gathered}
{[1,-1,-3,-3]=\left[\left(b_{3} d_{24}+b_{4} d_{23}\right) I_{2}+b_{3} d_{12} I_{1}\right.} \\
\left.+2 b_{3} A(1,1,-1,-1)\right] / d_{23} d_{34} \\
{[-1,-1,-3,-3]=\left(1 / d_{13}\right)\left(r_{23}^{-1}-r_{14}^{-1}+2 r_{34}^{-1}\right) I_{2}} \\
+\left(1 / d_{34}\right)\left(r_{23}^{-1}-r_{14}^{-1}\right) I_{1} \\
+\left(2 b_{3}^{2} / d_{13} d_{23} d_{34}\right) A(1,1,-1,-1) . \\
{[1,1,-1,-5]=\left[\left(d_{13} d_{24}+d_{14} d_{23}\right) I_{2}+d_{12} d_{13} I_{1}\right.} \\
\left.\quad+2 d_{14} A(1,1,1,-3)\right] / 3 d_{14} d_{43} . \\
{[1,-1,-1,-5]=\left(1 / 3 b_{4}\right)\left(r_{14}^{-1}-2 r_{24}^{-1}-2 r_{34}^{-1}\right) I_{2}} \\
-\left(b_{4} d_{12} d_{13} / 3 d_{14} d_{24} d_{34}\right) I_{1} \\
-\left(2 b_{4} / 3 d_{24} d_{34}\right) A(1,1,1,-3) . \\
{[-1,-1,-1,-5]=\left(2 / 3 d_{41}\right) \sum_{i=2}^{4} r_{i 4}^{-1} I_{2}} \\
+\left(b_{1} / d_{14}\right)^{2}\left(1-r_{12} r_{13} / 3 r_{24} r_{34}\right) I_{1} \\
-\left(2 b_{4}^{2} / 3 d_{14} d_{24} d_{34}\right) A(1,1,1,-3) .
\end{gathered}
$$

4. Reduction by Recurrence Relations. The integrals in Sections 2 and 3 are expressed in terms of $I_{1}, I_{2}, I_{3}$, and $I_{3}^{\prime}$ by using recurrence relations, most of which were proved in [4]. Let $e_{i}$ denote an $n$-tuple with 1 in the $i$ th place and 0's elsewhere (for example, $\left[p+2 e_{1}\right]=\left[p_{1}+2, p_{2}, \ldots, p_{n}\right]$ ). We denote $[4,(5.4)]$ by (Ai):

$$
\left(p_{1}+\cdots+p_{n}+2\right) b_{i}[p]=\sum_{j \neq i} p_{j} d_{j i}\left[p-2 e_{j}\right]+2 A\left(p+2 e_{i}\right)
$$

For concise reference we write $[4,(5.1)]$ in two forms:

$$
\begin{gathered}
d_{i j}[p]=b_{j}\left[p+2 e_{i}\right]-b_{i}\left[p+2 e_{j}\right] \\
b_{j}[p]=b_{i}\left[p-2 e_{i}+2 e_{j}\right]+d_{i j}\left[p-2 e_{i}\right] .
\end{gathered}
$$

Equation $(\mathrm{C} i j)$ is $(\mathrm{B} i j)$ with $p$ replaced by $p-2 e_{i}$. Finally, [4, (5.22)] implies $\sum\left(a_{i}+b_{i} t\right) d_{j k}=0$ and hence

$$
\sum\left[p+2 e_{i}\right] d_{j k}=0
$$

where $\sum$ denotes summation over cyclic permutations of $i, j, k$. Replacing $p$ by $p-2 e_{k}$, we see that

$$
d_{i j}[p]=d_{k j}\left[p+2 e_{i}-2 e_{k}\right]+d_{i k}\left[p+2 e_{j}-2 e_{k}\right] .
$$

We discuss first the reduction of the integrals in Section 3, because they will be used to reduce those in Section 2 and because the procedure differs at some points from that used in [4]. Equations (3.1) and (3.2) were proved in [4, §4]. Equation (3.3) obviously comes from (B14) and (3.4) from (D145). For (3.5) we first use (D342) to get

$$
d_{34}[1,1,-3,-3]=d_{24} I_{2}+d_{32}[1,-1,-3,-1]
$$


To reduce the last term, we put $[p]=[1,-1,-1,-1]$ in $(\mathrm{A} 1)$ and in $[4,(5.3)]$ to find, respectively,

$$
\begin{aligned}
& 0=d_{12}[1,-3,-1,-1]+d_{13}[1,-1,-3,-1]+d_{14} I_{2} \\
& +2 A(3,-1,-1,-1) \text {, } \\
& b_{1} I_{1}-b_{2}[1,-3,-1,-1]-b_{3}[1,-1,-3,-1]-b_{4} I_{2} \\
& =2 A(1,-1,-1,-1) \text {. }
\end{aligned}
$$

Elimination of $[1,-3,-1,-1]$ yields

$$
d_{32}[1,-1,-3,-1]=d_{24} I_{2}+d_{12} I_{1}+2 A(1,1,-1,-1),
$$

which we have simplified by using (4.8) and [4, (5.22)].

Equation (3.6) follows from (B23) and (3.7) from (B13). Equation (3.8) comes from putting $[p]=[1,1,-1,-3]$ in $(\mathrm{A} 1) ;(3.9)$ then follows from (B24) and (3.10) from (B14).

Equation (2.19) is a special case of (2.34). Equations (2.20) and (2.21) come from (C24) and (C14), respectively. To get (2.22) we use (A3) and evaluate $[-1,1,-1,-1]$ by $(\mathrm{C} 21)$. Equations $(2.23),(2.24),(2.25)$, and $(2.26)$ come from (C12), (C34), (C14), and (C34), respectively. For (2.27) we use (A4) and evaluate $[-1,1,1,-1]$ by $(\mathrm{C} 31)$ and $[1,-1,1,-1]$ by $(\mathrm{C} 32)$. For $(2.28)$ we use (A1) and evaluate $[1,-1,1,1]$ by $(\mathrm{C} 42)$ and $[1,1,-1,1]$ by (C43). Equations (2.29) to (2.33) follow in order from (C51), (C52), (C54), (C54), and (C51).

We shall prove (2.34) in Section 5. Equations (2.35) to (2.44) follow in order from (B15), (C25), (B15), (B45), (D452), (C35), (C15), (C15), (C35), and (C45). To get $(2.46)$ we put $[p]=[1,1,-1,-1,-2]$ in (A3) and evaluate $[-1,1,-1,-1,-2]$ by (C21). Equations (2.47) to (2.50) then follow in order from (B25), (C35), (B15), and (C45).

The formulas resulting from this procedure have often been simplified with the help of various identities. Besides $[4,(5.22)]$ we note

$$
\begin{gathered}
r_{i k}+r_{k j}=r_{i j}=-r_{j i}, \\
r_{i k}^{2}+r_{j k}^{2}-r_{i j}^{2}=2 r_{i k} r_{j k}, \\
d_{i j} A(p)=b_{j} A\left(p+2 e_{i}\right)-b_{i} A\left(p+2 e_{j}\right), \\
\sum A\left(p+2 e_{i}\right) d_{j k}=0
\end{gathered}
$$

where $\sum$ denotes summation over cyclic permutations of $i, j, k$. Equation (4.6) is obvious from (2.1), and (4.7) comes from squaring (4.6). Equation (4.8) follows from (2.6) and [4, (5.7)], and the proof of (4.9) is like that of (4.1).

5. The Fundamental Integral. Equations (2.13) and (2.14), with left sides identified in (2.17), were proved in [4, Section 4], initially with a restriction on the $a$ 's and $b$ 's that was later removed by using the analyticity of the $R$-function when each of its variables lies in the complex plane cut along the nonpositive real axis. The proof of (2.15) is similar except for a complication: The variables $W^{2}$ and $Q^{2}$ are sometimes real and negative. 
In this section we assume $x>y$ and $a_{i}+b_{i} t>0, y \leq t \leq x$, for $1 \leq i \leq 5$. From $[4,(4.1),(4.3)]$ and (1.3) we find the companion of $[4,(4.5),(4.6)]$ :

$$
\begin{aligned}
I_{3}=[1,-1,-1,-1,-2]= & \left(2 / 3 d_{15}\right)\left(d_{12} d_{13} d_{14}\right)^{-1 / 2} \\
& \cdot\left[R_{J}\left(z_{2}, \ldots, z_{5}\right)-R_{J}\left(z_{2}+\lambda, \ldots, z_{5}+\lambda\right)\right],
\end{aligned}
$$

where, for $i=2, \ldots, 5$,

$$
z_{i}=Y_{i}^{2} / Y_{1}^{2} d_{1 i}, \quad \lambda=(x-y) / X_{1}^{2} Y_{1}^{2}, \quad z_{i}+\lambda=X_{i}^{2} / X_{1}^{2} d_{1 i} .
$$

As in [4], this result depends on the temporary assumption that $-a_{1} / b_{1}$ is the first singularity of the integrand to the right of the interval of integration, which implies $d_{1 i}>0, i>1$, and thus guarantees that all variables of $R_{J}$ are positive. We now apply the addition theorem $[9,(8.11)]$,

$$
\begin{aligned}
& R_{J}\left(z_{2}, \ldots, z_{5}\right)=R_{J}\left(z_{2}+\lambda, \ldots, z_{5}+\lambda\right)+R_{J}\left(z_{2}+\mu, \ldots, z_{5}+\mu\right) \\
& +3 R_{C}(\gamma+\delta, \gamma), \\
& z_{i}+\mu=\lambda^{-2}\left\{\left[\left(z_{i}+\lambda\right) z_{j} z_{k}\right]^{1 / 2}+\left[z_{i}\left(z_{j}+\lambda\right)\left(z_{k}+\lambda\right)\right]^{1 / 2}\right\}^{2}, \\
& \gamma=z_{5}\left(z_{5}+\lambda\right)\left(z_{5}+\mu\right), \quad \delta=\left(z_{2}-z_{5}\right)\left(z_{3}-z_{5}\right)\left(z_{4}-z_{5}\right) .
\end{aligned}
$$

In (5.4), $i, j, k$ is any permutation of $2,3,4$. Equation (5.1) becomes

$$
I_{3}=\left(2 / 3 d_{15}\right)\left(d_{12} d_{13} d_{14}\right)^{-1 / 2}\left[R_{J}\left(z_{2}+\mu, \ldots, z_{5}+\mu\right)+3 R_{C}(\gamma+\delta, \gamma)\right] .
$$

From (5.4) and (5.2) we find, for $i=2,3,4$,

$$
\begin{gathered}
z_{i}+\mu=U_{1 i}^{2} / d_{12} d_{13} d_{14}, \\
z_{5}+\mu=\left(z_{i}+\mu\right)+\left(z_{5}-z_{i}\right), \\
z_{5}-z_{i}=\left(d_{1 i} Y_{5}^{2}-d_{15} Y_{i}^{2}\right) / d_{15} d_{1 i} Y_{1}^{2}=-d_{i 5} / d_{15} d_{1 i},
\end{gathered}
$$

where $[4,(5.23)]$ is used in the last step. It follows that

$$
\begin{gathered}
z_{5}+\mu=W^{2} / d_{12} d_{13} d_{14}, \\
\gamma=Q^{2} / d_{12} d_{13} d_{14} d_{15}^{2}, \quad \gamma+\delta=P^{2} / d_{12} d_{13} d_{14} d_{15}^{2},
\end{gathered}
$$

where $W^{2}, P^{2}$, and $Q^{2}$ are defined by (2.4) and (2.5). Homogeneity of $R_{J}$ and $R_{C}$ [see (1.4) and (1.6)] now yields (2.15) from (5.6).

Since $z_{2}, z_{3}, z_{4}, \lambda$ are positive by (5.2), expansion of the right-hand side of (5.4) shows that $\mu>0$. Since $z_{5}>0$ by (5.2), it follows by (5.8) that $W^{2}>0$ and by (2.5) that $Q^{2}>0$. Equation (2.8) shows that $P^{2}>0$. Thus the $R$-functions in (5.6) and (2.15) are well defined when the temporary assumption about $-a_{1} / b_{1}$ is satisfied. When it is not, the functions in (2.15) remain well defined if $W^{2}$ and $Q^{2}$, which have the same signs by $(2.5)$, are positive.

To examine the sign of $W^{2}$, we let $w=X_{1}^{2}=a_{1}+b_{1} x$ and allow $w$ to vary while fixing $x$ and $y$ and $X_{i}>0,2 \leq i \leq 5$, and $Y_{i}>0,1 \leq i \leq 5$. If we define

$$
w_{i}=X_{i}^{2} Y_{1}^{2} / Y_{i}^{2},
$$

then $w_{2}, \ldots, w_{5}$ are fixed positive quantities, $w=w_{1}$ is a positive variable, and $d_{1 i}$ is a linear function of $w$ because

$$
(x-y) d_{1 i}=X_{i}^{2} Y_{1}^{2}-Y_{i}^{2} X_{1}^{2}=Y_{i}^{2}\left(w_{i}-w\right) .
$$


We shall want also, for $i=2,3,4$,

$$
(x-y) d_{i 5}=X_{5}^{2} Y_{i}^{2}-Y_{5}^{2} X_{i}^{2}=\left(Y_{i} Y_{5} / Y_{1}\right)^{2}\left(w_{5}-w_{i}\right) .
$$

Since $Q^{2}$ and $W^{2}$ are positive by (2.5) if $d_{25} d_{35} d_{45}=0$, we may assume that $w_{5}$ is not equal to $w_{2}, w_{3}$, or $w_{4}$.

The graph of $a_{1}+b_{1} t$ has the fixed positive ordinate $Y_{1}^{2}$ at $t=y$, the variable positive ordinate $w$ at $t=x$, and the intercept $-a_{1} / b_{1}$ on the $t$-axis. As $w$ runs through all positive real values, this intercept takes all values outside the fixed interval $[y, x]$ of integration. When $w=w_{i}$ for some $i>1$, the intercept coincides with the fixed intercept $-a_{i} / b_{i}$ of the graph of $a_{i}+b_{i} t$. The temporary assumption about $-a_{1} / b_{1}$ means that $0<w<w_{i}, 2 \leq i \leq 5$.

We find from (2.4) and (2.3) that

$$
\begin{gathered}
W^{2}=\left[Y_{2} Y_{3} Y_{4} / Y_{1}(x-y)\right]^{2} f(w) \\
f(w)=\left[\left(w w_{2}\right)^{1 / 2}+\left(w_{3} w_{4}\right)^{1 / 2}\right]^{2}-\left(w_{3}-w\right)\left(w_{4}-w\right)\left(w_{5}-w_{2}\right) /\left(w_{5}-w\right) .
\end{gathered}
$$

This is positive when $w$ equals $w_{2}, w_{3}$, or $w_{4}$, but it changes sign as $w$ goes through the singularity at $w_{5}$. Writing $\varsigma$ for the positive square root $w^{1 / 2}=X_{1}$, we find

$$
\begin{aligned}
\left(w-w_{5}\right) f(w)= & w_{5} \varsigma^{4}+2\left(w_{2} w_{3} w_{4}\right)^{1 / 2} \varsigma^{3} \\
& +\left[w_{2} w_{3}+w_{2} w_{4}+w_{3} w_{4}-w_{5}\left(w_{2}+w_{3}+w_{4}\right)\right] \varsigma^{2} \\
& -2 w_{5}\left(w_{2} w_{3} w_{4}\right)^{1 / 2} \varsigma-w_{2} w_{3} w_{4} .
\end{aligned}
$$

By Descartes' rule of signs the polynomial in $\varsigma$ has exactly one positive zero, say $\varsigma_{0}=w_{0}^{1 / 2}$. Thus $f(w)$ changes sign exactly twice, at $w_{0}$ and $w_{5}$, as $w$ goes through all positive values. Since $f$ is positive at 0 and $\infty$, it must be negative in the open interval with endpoints $w_{0}$ and $w_{5}$, either of which may be the greater. This interval cannot contain $w_{2}, w_{3}$, or $w_{4}$ since $f$ is positive at those points.

We shall now establish (2.15) for all $w>0$ by analytic continuation. We cut the $w$-plane along the nonpositive real axis and make a second cut between $w_{0}$ and $w_{5}$ on the positive real axis. The corresponding region of the $\zeta$-plane is the open right half-plane with a cut between $w_{0}^{1 / 2}$ and $w_{5}^{1 / 2}$ on the positive real axis. The coefficient of $R_{J}$ in (2.15) and the variables of both $R_{J}$ and $R_{C}$ are analytic functions of $w$ in the cut $w$-plane by (2.3), (2.4), (2.5), (5.11), and (5.12). We recall that an $R$-function is analytic when each of its variables lies in the complex plane cut along the nonpositive real axis $[2,(6.8-6)$, Theorem 6.8-1]. Therefore, if we can show that none of the variables can be real and nonpositive when $w$ is in the cut $w$-plane, it will follow that $R_{J}, R_{C}$, and the right-hand side of (2.15) are analytic.

Since $U_{12}, U_{13}$, and $U_{14}$ are linear functions of $\zeta$ with positive coefficients by (2.3), they have positive real parts when $\varsigma$ does, and their squares cannot be real and nonpositive. A similar remark, with $\varsigma$ replaced by $1 / \varsigma$, applies to $P^{2}$ by $(2.8)$. It remains to consider $W^{2}$ and $Q^{2}$.

Now $W^{2}$ is real and nonpositive if and only if $f(w)+r=0$ for some $r \geq 0$. The quantity

$$
\left(w-w_{5}\right)[f(w)+r]=\left(w-w_{5}\right) f(w)+r \varsigma^{2}-r w_{5}
$$

is a quartic polynomial in $\zeta$ whose coefficients, like those of (5.14), have the signs

$$
+,+, \pm,-,-
$$


By Descartes' rule of signs there is exactly one positive zero, which must lie on the cut between $w_{0}^{1 / 2}$ and $w_{5}^{1 / 2}$ where $f(w)$ takes every nonpositive real value. Either the other three zeros are real and negative or else one is negative and the other two are conjugate complex. In the latter case the polynomial must have the form

$$
\begin{aligned}
w_{5}(\varsigma & -\xi)(\varsigma+\eta)(\varsigma-\alpha-i \beta)(\varsigma-\alpha+i \beta) \\
& =w_{5}\left[\varsigma^{2}+(\eta-\xi) \varsigma-\xi \eta\right]\left[\varsigma^{2}-2 \alpha \varsigma+\alpha^{2}+\beta^{2}\right],
\end{aligned}
$$

where $\xi$ and $\eta$ are positive and $\alpha$ and $\beta$ are real. The coefficients of $\varsigma^{3}$ and $\zeta$ have the signs required by (5.16) if and only if

$$
\eta-\xi-2 \alpha>0, \quad\left(\alpha^{2}+\beta^{2}\right)(\eta-\xi)+2 \alpha \xi \eta<0,
$$

which imply $2 \alpha\left(\alpha^{2}+\beta^{2}+\xi \eta\right)<0$ and hence $\alpha<0$. Thus the conjugate complex roots have negative real part. We conclude that a quartic polynomial whose coefficients have the signs (5.16) has one real positive zero and three zeros in the open left half-plane. Thus no zero of (5.15) lies in the cut right-half $\zeta$-plane, and $W^{2}$ cannot be real and nonpositive at any point in the cut $w$-plane.

By (2.5) and (5.13), $Q^{2}$ is a positive multiple of $f(w) / w$. Since $w \neq 0$ in the cut plane, $Q^{2}$ is real and nonpositive if and only if $f(w)+r w=0$ for some $r \geq 0$. The quantity

$$
\left(w-w_{5}\right)[f(w)+r w]=\left(w-w_{5}\right) f(w)+r \varsigma^{4}-w_{5} r \varsigma^{2}
$$

is a quartic polynomial in $\varsigma$ whose coefficients, like those of (5.14), have the signs (5.16). Thus $Q^{2}$, like $W^{2}$, cannot be real and nonpositive at any point of the cut plane. This completes the proof that the right-hand side of (2.15) is analytic in $w$ on the cut $w$-plane.

The left side of $(2.15)$ is defined by (1.3), which can be rewritten in the form of $[4,(4.12)]$ :

$$
\begin{gathered}
I_{3}=[1,-1,-1,-1,-2]=(x-y)\left(Y_{1} / Y_{2} Y_{3} Y_{4} Y_{5}^{2}\right) \\
\cdot R_{-1}\left(\frac{-1}{2}, \frac{1}{2}, \frac{1}{2}, \frac{1}{2}, 1 ; \frac{w}{Y_{1}^{2}}, \frac{X_{2}^{2}}{Y_{2}^{2}}, \ldots, \frac{X_{5}^{2}}{Y_{5}^{2}}\right) .
\end{gathered}
$$

This is analytic in the $w$-plane cut along the nonpositive real axis, even without the second cut from $w_{0}$ to $w_{5}$. Since (2.15) is known to hold if $0<w<w_{i}, 2 \leq i \leq 5$, it holds throughout the twice-cut $w$-plane by the permanence of functional relations. Each of the two terms on the right-hand side of (2.15) is discontinuous across the second cut, but the discontinuities must cancel (as can be verified explicitly) because the left side is continuous. Hence the second cut is unnecessary and can be removed after defining $R_{J}$ and $R_{C}$ on the cut so as to make the right-hand side continuous. A suitable definition for each is its Cauchy principal value, which is the arithmetic average of its values on the upper and lower edges of the cut. With this definition, (2.15) holds on the $w$-plane cut only along the nonpositive real axis. In particular it holds for all positive values of $w$, which was to be proved.

At each endpoint of the second cut the Cauchy principal value is to be interpreted as a limit of values off the cut or of principal values on the cut. The limit is easy to evaluate at the end where $w=w_{5}$, since this implies by (5.11) that $d_{15}$ vanishes, $a_{5}+b_{5} t$ is proportional to $a_{1}+b_{1} t$, and $[1,-1,-1,-1,-2]$ reduces to $\left(a_{1} / a_{5}\right)[-1,-1,-1,-1]$. 
6. Cauchy Principal Values. According to Section 5 , the evaluation of $I_{3}$ may involve Cauchy principal values of both $R_{J}$ and $R_{C}$, even though $a_{5}+b_{5} t$ is positive on the closed interval of integration. We shall prove now that formulas (2.34) to (2.44) are still valid if $a_{5}+b_{5} t$ changes sign in the open interval of integration. If the left side of each formula is taken to be a Cauchy principal value, then $I_{3}$ as defined by (2.15) involves the Cauchy principal value of either $R_{J}$ or $R_{C}$ but not both, since $W^{2}$ and $Q^{2}$ have opposite signs by (2.5) when $X_{5}^{2} Y_{5}^{2}<0$.

It suffices to consider (2.34), since the proofs of (2.35) to (2.44) by recurrence relations $(\mathrm{B} i j),(\mathrm{C} i j)$, and (Dijk) do not depend on the sign of $a_{5}+b_{5} t$. We shall assume $a_{5}+b_{5} x<0<a_{5}+b_{5} y$, but the proof with $x$ and $y$ interchanged is similar. The method is much like that in Section 5 .

We fix $x$ and $y$ and $X_{i}>0,1 \leq i \leq 4$ and $Y_{i}>0,1 \leq i \leq 5$, while allowing $X_{5}^{2}=a_{5}+b_{5} x$ to vary in the complex plane. By $(5.10), w_{1}, \ldots, w_{4}$ are fixed positive quantities while $w_{5}=X_{5}^{2} Y_{1}^{2} / Y_{5}^{2}$ is variable. Since $w$ is an abbreviation for $w_{1}$, we see from (5.11) that the coefficient of $R_{J}$ in (2.15) is analytic except for a pole at $w_{1}$. The first three arguments of $R_{J}$ are fixed and positive, but it follows from (5.13) that the fourth argument has the form

$$
W^{2}=A+\frac{B}{w_{5}-w_{1}},
$$

where $A$ and $B$ are real and independent of $w_{5}$. A little algebra shows that $A>0$ if $B=0$, and hence $W^{2}$ cannot be real and nonpositive unless ${ }^{\prime}{ }_{5}$ is real. We cut the $w_{5}$-plane along the nonpositive real axis and also along every interval of the positive real axis where $W^{2} \leq 0$. Unless $B=0$, one of these intervals will have the pole at $w_{1}$ as one endpoint, and so the cut plane will in all cases include an open interval of the positive real axis, where $X_{5}^{2}>0$ and (2.15) is valid by Section 5 . In the cut plane, $W^{2}$ is analytic in $w_{5}$ and cannot be real and nonpositive. Hence, the first term on the right-hand side of $(2.15)$ is analytic in $w_{5}$ on the cut plane.

We rewrite the second term, using the homogeneity of $R_{C}$, as

$$
2 w_{5}^{-1 / 2} R_{C}\left(\left(P w_{5}^{-1 / 2}\right)^{2}, Q^{2} / w_{5}\right)
$$

Equation (2.8) shows that

$$
P w_{5}^{-1 / 2}=M w_{5}^{-1 / 2}+N w_{5}^{1 / 2}
$$

where $M$ and $N$ are positive and independent of $w_{5}$. If $w_{5}$ is in the cut plane, both terms on the right-hand side of (6.3) lie in the open right half-plane, and hence $\left(P w_{5}^{-1 / 2}\right)^{2}$ cannot be real and nonpositive. Neither can $Q^{2} / w_{5}$, since it is a positive multiple of $W^{2}$ by (2.5). Thus, the second term on the right-hand side of (2.15) also is analytic in $w_{5}$ on the cut plane.

Finally, the left side of $(2.15)$ is analytic in $w_{5}$ on the cut plane because the first four arguments of $R_{-1}$ in (5.20) are fixed and positive while the last argument is a positive multiple of $w_{5}$. Since the cut plane contains an open interval of the positive real axis and (2.15) holds on that interval, it holds everywhere in the cut plane by the permanence of functional relations. The equality clearly persists if each term in the equation is replaced by the arithmetic average of its values on the upper and lower edges of the cut along the negative real axis. On the left side this average is the Cauchy principal value of $I_{3}$. On the right-hand side, either $R_{J}$ or $R_{C}$ takes 
its Cauchy principal value, but since $Q^{2}$ and $W^{2}$ have opposite signs when $w_{5}$ is negative, one of the two functions will be continuous across the cut.

A numerical example in which $I_{3}$ and $R_{C}$ take their principal values is given at the end of the Supplement.

Ames Laboratory and Department of Mathematics Iowa State University

Ames, Iowa 50011

1. P. F. BYRD \& M. D. FRIEDMAN, Handbook of Elliptic Integrals for Engineers and Scientists, 2nd ed., Springer-Verlag, New York, 1971.

2. B. C. CARLSON, Special Functions of Applied Mathematics, Academic Press, New York, 1977.

3. B. C. CARLSON, "Computing elliptic integrals by duplication," Numer. Math., v. 33, 1979, pp. 1-16.

4. B. C. CARLSON, "A table of elliptic integrals of the second kind," Math. Comp., v. 49, 1987, pp. 595-606. (Supplement, ibid., S13-S17.)

5. B. C. CARLSON \& Elaine M. Notis, "AlgORITHM 577, Algorithms for incomplete elliptic integrals," ACM Trans. Math. Software, v. 7, 1981, pp. 398-403.

6. G. FUBINI, "Nuovo metodo per lo studio e per il calcolo delle funzioni trascendenti elementari," Period. Mat., v. 12, 1897, pp. 169-178.

7. I. S. GRadshteyn \& I. M. RYZhiK, Table of Integrals, Series, and Products, Academic Press, New York, 1980.

8. A. P. PRUdNikov, YU. A. BRYChKov \& O. I. MARICHEV, Integrals and Series, Vol. 1, Gordon and Breach, New York, 1986.

9. D. G. Zill \& B. C. CARLSON, "Symmetric elliptic integrals of the third kind," Math. Comp., v. 24, 1970, pp. 199-214. 


\title{
Supplement to A Table of Elliptic Integrals of the Third Kind
}

\author{
By B. C. Carlson
}

This supplement contains Fortran codes for the functions $R_{C}(x, y)$ and $R_{J}(x, y, z, p)$. If $y$ or $p$ is negative, the cauchy principal value is computed. The codes are followed by some numerical values that were used to check the formulas in sections 2 and 3 .

DOUBLE PRECISION FUNCTION RC(X,Y,ERRTOL, IERR)

THIS FUNCTION SUBROUTINE COMPUTES THE ELEMENTARY INTEGRAL $\operatorname{RC}(\mathrm{X}, \mathrm{Y})=$ INTEGRAL FROM ZERO TO INFINITY OF

$$
(1 / 2)(T+X)^{-1 / 2}(T+Y)^{-1} D T \text {, }
$$

WHERE $X$ IS NONNEGATIVE AND $Y$ IS NONZERO. IF $Y$ IS NEGATIVE, THE CAUCHY PRINCIPAL VALUE IS COMPUTED BY USING A PRELIMINARY TRANSFORMATION TO MAKE Y POSITIVE; SEE EOUATION (2.12) OF THE SECOND REFERENCE BELOW. WHEN Y IS POSITIVE, THE DUPLICATION THEOREM IS ITERATED UNTIL THE VARIABLES ARE NEARLY EQUAL, AND THE FUNCTION IS THEN EXPANDED IN TAYLOR SERIES TO FIFTH ORDER. LOGARITHMIC, INVERSE CIRCULAR, AND INVERSE HYPERBOLIC FUNCTIONS ARE EXPRESSED IN TERMS OF RC BY EQUATIONS (4.9)-(4.13) OF THE SECOND REFERENCE BELOW. REFERENCES: B. C. CARLSON AND E. M. NOTIS, ALGORITHMS FOR INCOMPLETE ELLIPTIC INTEGRALS, ACM TRANSACTIONS ON MATHEMATICAL SOFTWARE, $7(1981), 398-403$; B. C. CARLSON, COMPUTING ELLIPTIC INTEgRALS By DUPLiCATION, NUMER. MATH. 33 (1979), $1-16$.

AUTHORS: B. C. CARLSON AND ELAINE M. NOTIS, AMES LABORATORYDOE, IOWA STATE UNIVERSITY, AMES, IA 50011, AND R. L. PEXTON, LAWRENCE LIVERMORE NATIONAL LABORATORY, LIVERMORE, CA 94550. AUG. 1, 1979, REVISED SEPT. 1, 1987.

CHECK VALUES: $\mathrm{RC}(0,1 / 4)=\mathrm{RC}(1 / 16,1 / 8)=\mathrm{PI}$, $\operatorname{RC}(9 / 4,2)=\operatorname{LN}(2)$ $\operatorname{RC}(1 / 4,-2)=\operatorname{LN}(2) / 3$

CHECK BY ADDITION THEOREM: $R C(X, X+Z)+R C(Y, Y+Z)=R C(0, Z)$ WHERE $X, Y$, AND $Z$ ARE POSITIVE AND $X * Y=Z * Z$. 

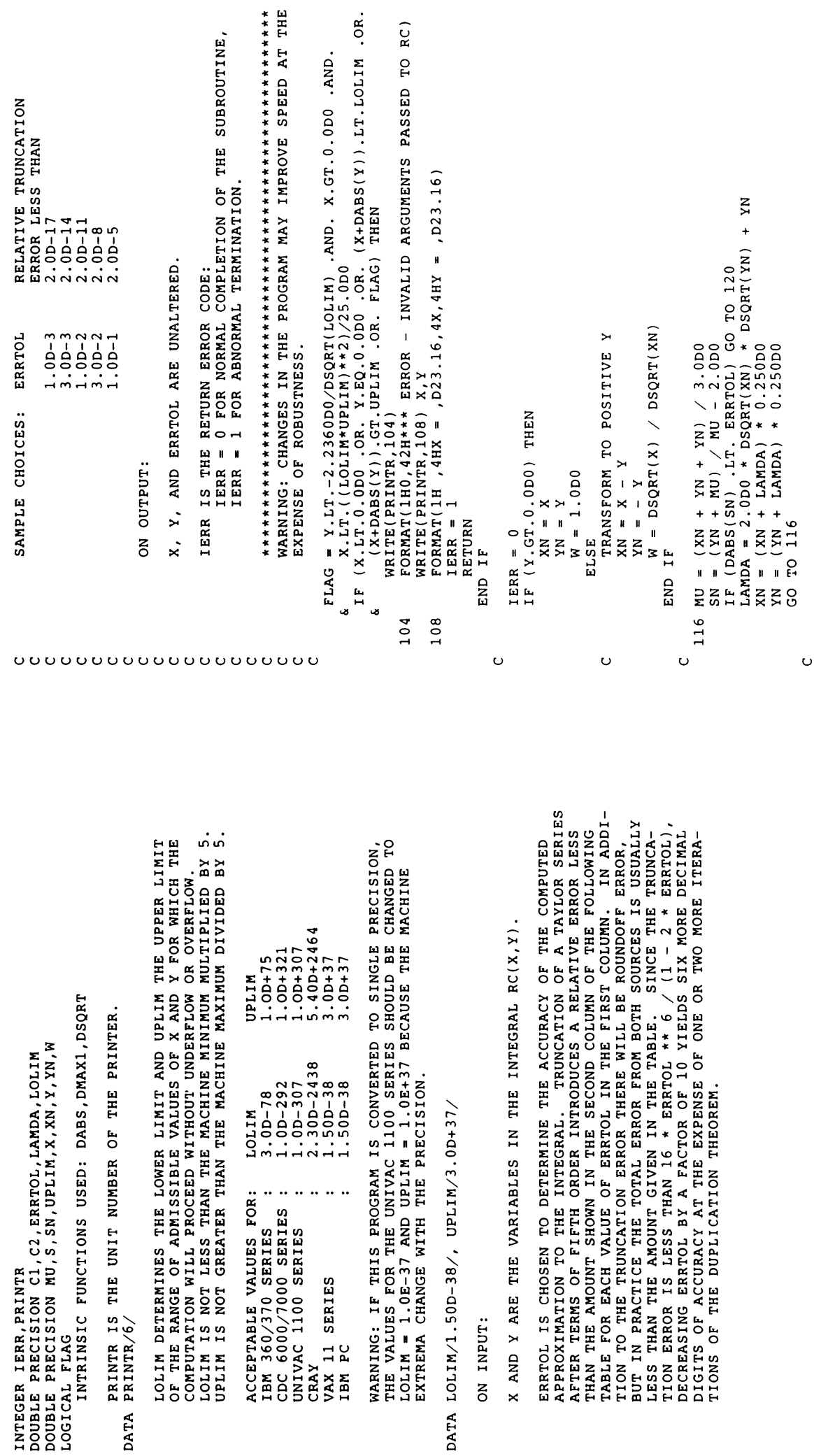

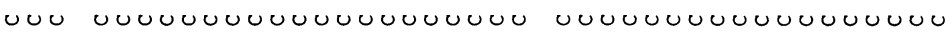



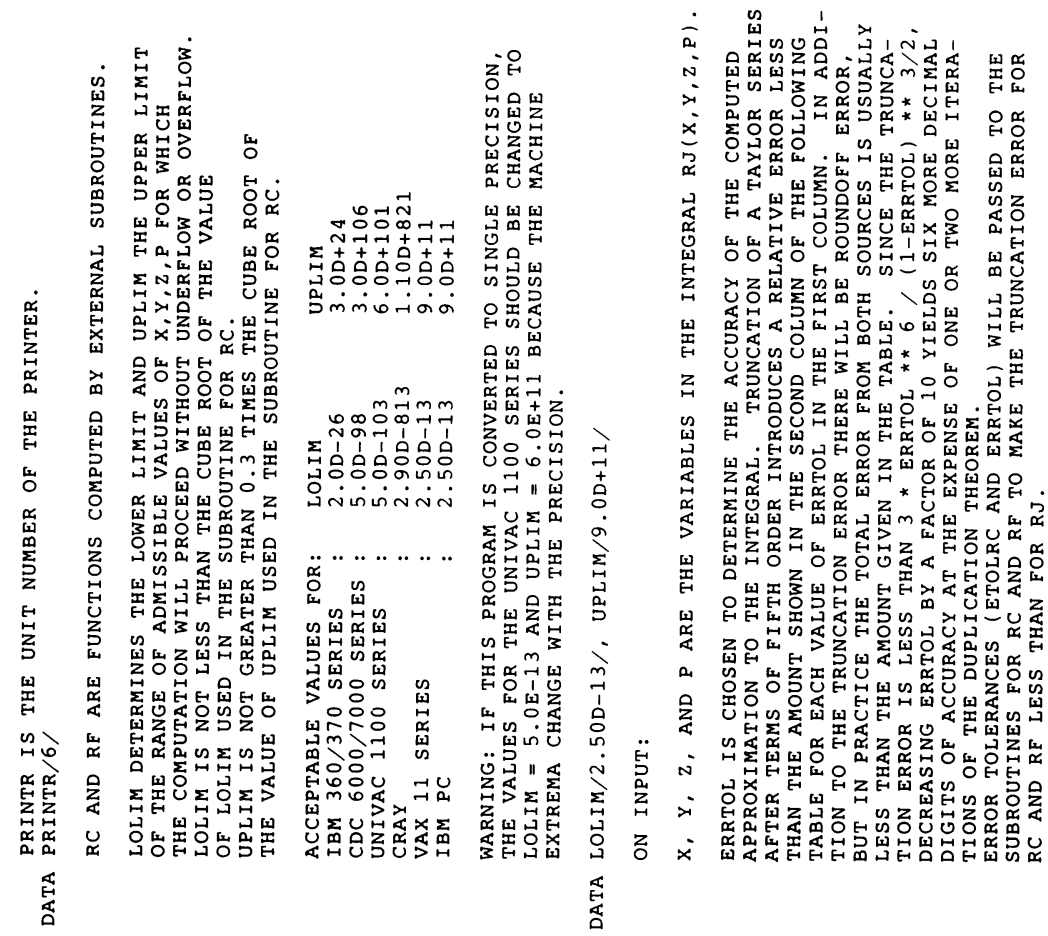

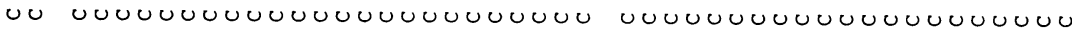

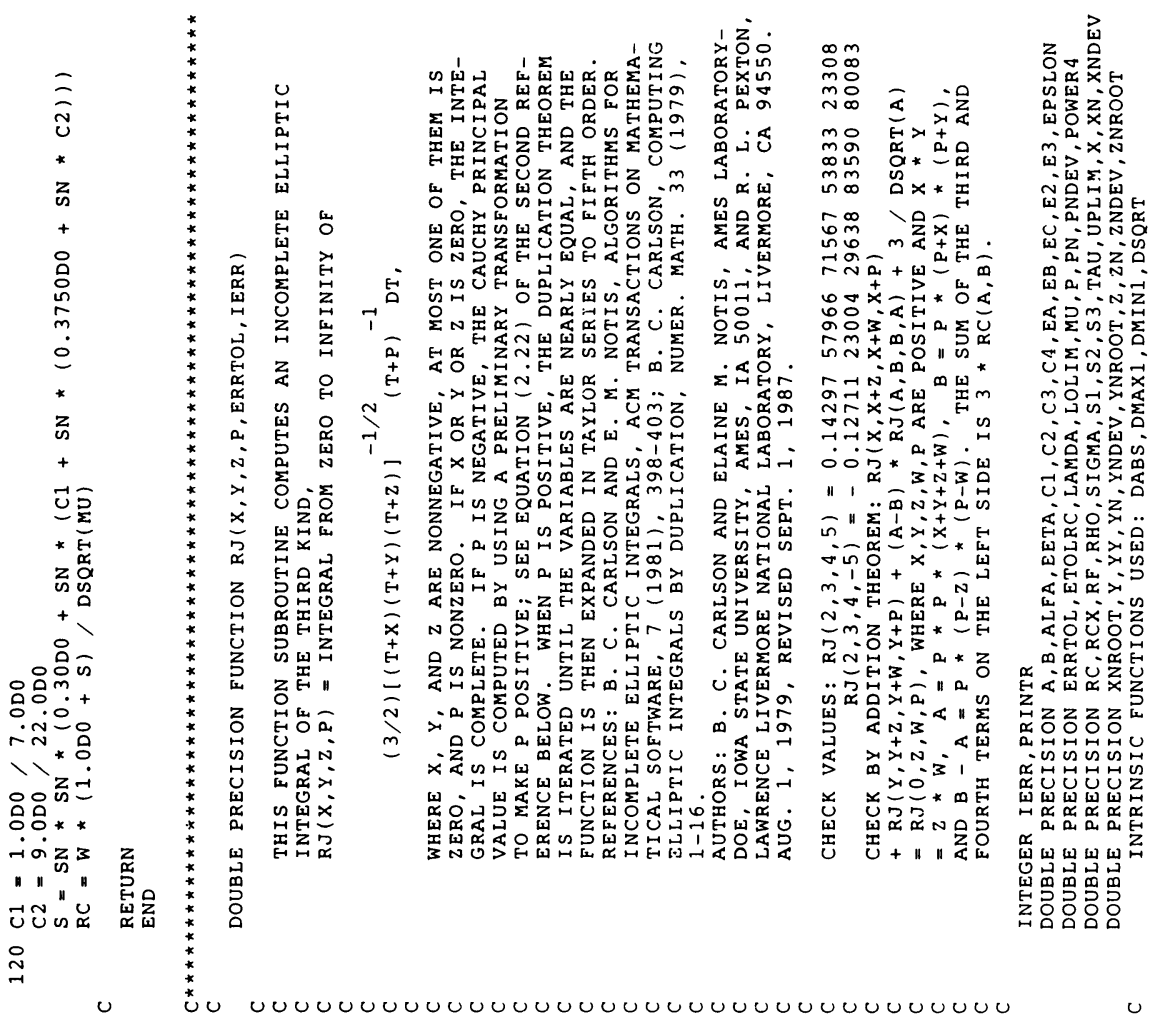



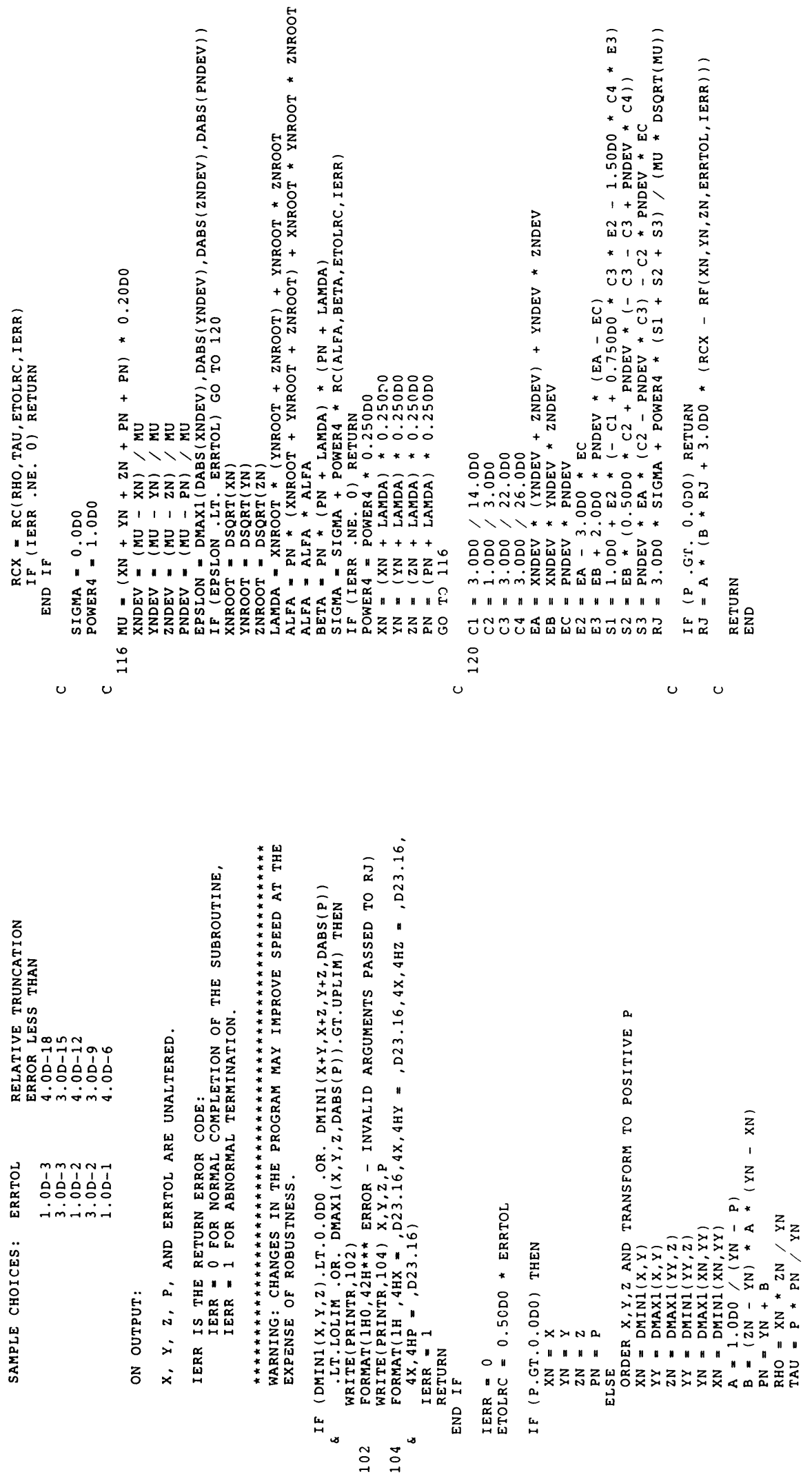

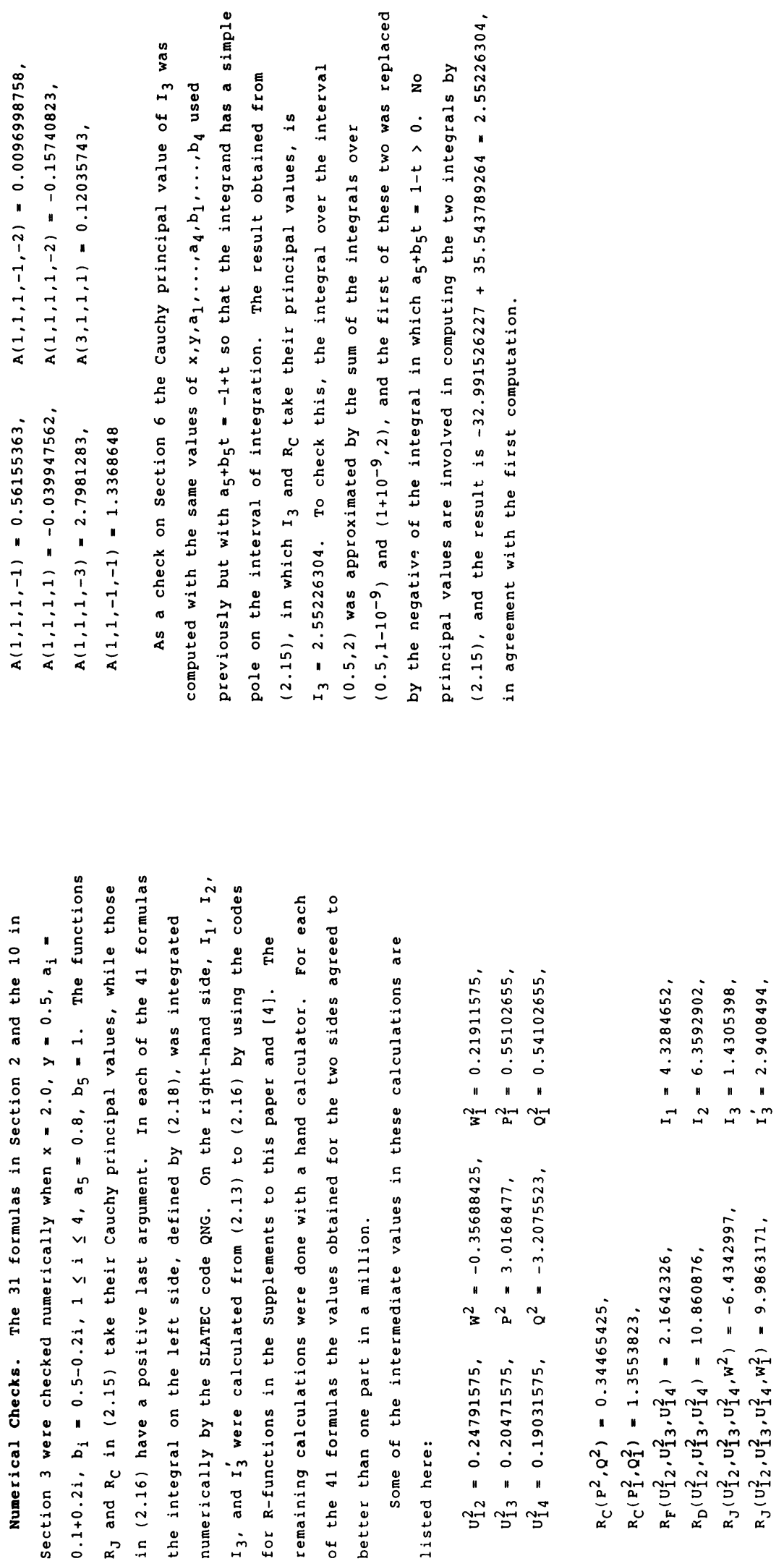